\title{
THE STELLAR TEMPERATURE SCALE FROM ANGULAR DIAMETERS AND FLUX DISTRIBUTIONS
}

\author{
A.J.BOOTH \\ University of Sydney, \\ School of Physics A28, NSW 2006, Australia
}

\begin{abstract}
The current situation for the measurement of effective temperatures from interferometrically determined angular diameters and spectrophometry in the UV, visible and IR is considered. Accuracies and reliabilities of the resulting temperatures are assessed and coverage of stellar types is discussed.
\end{abstract}

\section{Introduction}

The effective temperature, $T_{e}$, of a star (or equivalently the total emergent flux, $F$ ) can be determined from two quantities that are in principle directly observable: the angular diameter $\theta$, and the total flux received at the Earth $F_{E}$. The definition of effective temperature is

$$
\sigma T_{e}=F,
$$

and, in the absence of interstellar absorption,

$$
F=\frac{4}{\theta^{2}} F_{E}
$$

hence

$$
T_{e}=\frac{1}{\sigma} \frac{4}{\theta^{2}} F_{E}
$$

Angular sizes can be determined from speckle interferometry (for very large $\theta$ stars), lunar occultation, or long baseline optical interferometry. This paper will be concerned with the last (see Richichi, this volume, for effective temperatures determined by lunar occultation).

Total fluxes must be found by integrating the observed monochromatic stellar flux from $0 \leq \lambda \leq \infty$. This requires spectrophotometric and calibrating photometric observations. A distinction should be noted with the 
Infra-Red Flux Method, IRFM, (Megessier, this volume), which requires only a ratio of fluxes (total to infra-red) whereas we require an absolute flux.

This paper will describe the present situation with particular regard to coverage, and accuracy and reliability. It will also discuss only "normal", and single stars. As yet very few "special" stars (eg. pulsating stars, shell stars), or stars in binaries have measured angular sizes.

\section{Coverage}

At present the number of stars with accurate, interferometrically determined temperatures are rather few, and this number is limited by the available angular size measurements. In principle the flux is more easily measurable than $\theta$ in that it does not require specialised instruments or observing techniques.

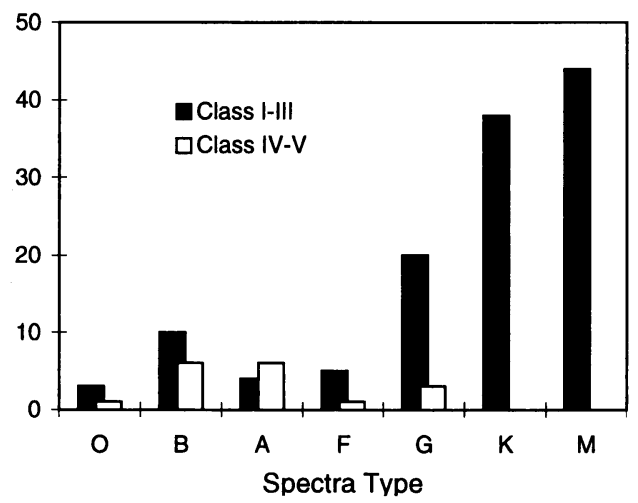

Figure 1. Histogram of measured angular sizes as a function of spectral type and luminosity class.

Davis (this volume) gives a review of the available angular diameters and their accuracies. Fig. 1 summarises these. Although Fig. 1 might at first suggest quite extensive coverage a few points should be noted. The figure includes all measurements with uncertainties $\lesssim 10 \%$. if only high accuracy values are accepted $(\lesssim 3-4 \%)$ the numbers drop by about a factor of two. In the $\mathrm{O}, \mathrm{B}, \mathrm{A}$, range of type the measurements are mostly from the Narrabri Intensity Interferometer (Code et al., 1976). It is a tribute to Hanbury Brown and his co-workers that these measurements made some 20 years ago still form the basis of the temperature scale for hot stars. However, both the angular size and flux measurement accuracies can now be improved for these stars. Of the other stars in Fig. 1 most are from the Mk.III interferometer (Hutter et al., 1989), with some from the IOTA (Dyck 
et al., 1996) or I2T interferometers (Di Benedetto \& Rabbia, 1987). Of the Mk.III angular diameters few have been turned into effective temperatures (Mozurkewich, private communication), though the flux measurements to do so are often available. Further, there are in general very few measurements for dwarfs, and in particular none for types $\mathrm{K}$ and $\mathrm{M}$. Indeed the only direct $T_{e}$ measurements for type MV comes from two eclipsing binary systems (Habets \& Heintze, 1981). So the main sequence in the K, M region has a very poorly defined temperature scale. Finally, there is a particular lack of any measurements for types $\mathrm{A}$ and $\mathrm{F}$.

\section{Accuracy and Reliability}

Since $T_{e} \propto \theta^{-2}$ and $T_{e} \propto F_{E}{ }^{4}$, the error in $T_{e}$ is insensitive to errors in $\theta$ or $F_{E}$. Roughly speaking, the formal error in $T_{e}, \sigma\left(T_{e}\right)$, is given by

$$
\sigma\left(T_{e}\right) \propto \frac{1}{2} \sigma(\theta) \text { and } \sigma\left(T_{e}\right) \propto \frac{1}{4} \sigma\left(F_{E}\right)
$$

Too much can be read into this result, however, as $T_{e}$ is an many ways an unphysical quantity, and should really be thought of as a label for a particular set of atmospheric conditions. The physical quantity is $F\left(\propto T_{e}^{4}\right)$ which does not have these desirable dependencies of its accuracy. Having said this, I shall continue to use $T_{e}$ here. A good target accuracy for $T_{e}$ determinations is $1 \%$, which for example matches the best atomic data available for abundance determinations, and the best determinations of $\log (g)$ (Maxted, this volume). Hence, the target accuracy of $\theta$ should be $\lesssim 2 \%$ and of $F_{E} \lesssim 4 \%$.

As indicated above, the situation for the accuracy of $\theta$ measurements is quite good. Angular sizes with formal accuracy $\lesssim 2 \%$ (Davis, this volume) can be found quite readily, at least for a limited number of stars. However, the quoted accuracies are usually the internal consistency errors. Interferometric measurements are very sensitive to calibration uncertainty and there is some evidence of systematic errors of up to $10 \%$, for example between IOTA and I2T values (Dyck et al., 1996). Both the coverage problem and this calibration uncertainty are due to the limited number of currently operating interferometers and their restricted baselines. When the next generation of interferometers (eg. CHARA, NPOI, SUSI, VLTI) come into operation this situation is likely to be greatly improved (eg. Booth et al., 1997 for improvement in coverage).

The situation for the accuracy of the flux measurements is less satisfactory and it turns out that $F_{E}$ measurements limit the accuracy of the present $T_{e}$ determinations. It is useful to break up the $F_{E}$ measurements into 4 or 5 different wavelength regimes: 


\subsection{FAR UV FLUXES}

In the region $\lambda \lesssim 100 \mathrm{~nm}$ interstellar absorption blocks most of the flux, so direct measurements are largely impossible. Models must be used, and their accuracy is uncertain, one may guess at $20 \%$. This region is very important for the hottest stars and makes the measurement of their $T_{e}$ particularly difficult.

\subsection{UV FLUXES}

The region $100 \mathrm{~nm} \lesssim \lambda \lesssim 350 \mathrm{~nm}$ can be sampled by IUE or HST measurements. Recently the calibration in this region has been improved using white dwarf model atmospheres, but a good direct calibration would be more satisfactory to avoid modeling errors (Kruk, this volume). Internal consistency errors are typically $2-7 \%$ on well observed IUE stars, so, including a contribution for calibration uncertainty, a reasonable flux uncertainty in this region might be taken as $5 \%$.

\subsection{VISIBLE FLUXES}

Megessier (this volume) reviews the current calibration situation, with the absolute calibration of Vega being secure at about $0.7 \%$. Careful spectrophotometry can give internal consistency at about the $1 \%$ level (eg. Petford et al., 1988), though there are systematic differences at the 1$2 \%$ level between observers. A reasonable level of uncertainty in the range $350 \mathrm{~nm} \lesssim \lambda \lesssim 1 \mu \mathrm{m}$ might thus be $1-2 \%$.

\subsection{INFRA-RED AND FAR INFRA-RED FLUXES}

The situation in the infra-red is less clear (Megessier, this volume). The direct calibration of Vega for $1 \mu \mathrm{m} \lesssim \lambda \lesssim 5 \mu \mathrm{m}$ has internal consistency at the $3 \%$ level, but differs from models at the $7 \%$ level. The implication from the application of the IRFM is that the models are correct (Blackwell et al., 1991). A good test of this would be to compare IRFM deduced angular sizes with those found from interferometry. At longer wavelengths IRAS flux measurements can be used, but their calibration rests on model atmosphere colours (Cohen et al., 1992) and consequently are prone to unknown levels of systematic uncertainty. Fortunately, this region makes only a small contribution for most stars, and can often be well approximated by a black body function. Until these questions are resolved, a level for the flux accuracy in the infra-red could be about $5 \%$, but this may be optimistic.

Fig. 2 shows how these errors combine to produce a total error for $T_{e}$. This graph is based on black body curves not atmosphere models, but shows 


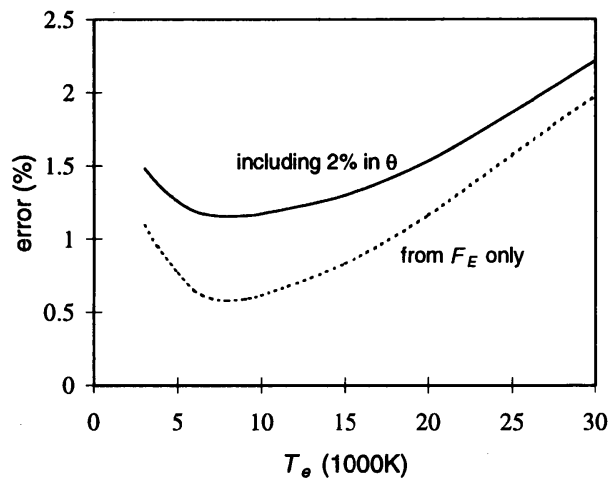

Figure 2. Total uncertainty in $T_{e}$ due to flux and angular size errors.

the general trends. Clearly we are well placed with regard to the types A to $\mathrm{G}$, but for hotter and cooler stars the calibrations of the infra-red and UV fluxes, respectively, need to be better defined. Some caution is called for, however, as many of the calibration errors are likely to be systematic rather than random. As such they are not improved by averaging, and further I may be underestimating their effect here by taking as a "typical" error the middle of the estimated range.

\section{Potential Problems}

There are also several caveats that must be placed on the above analysis, where systematic errors may make Fig. 2 overly optimistic.

\subsection{INTERSTELLAR ABSORPTION}

It is particularly difficult to determine an accurate value for $A_{V}$ for a given star. Different methods tend to give quite different results when stars are more than about $100 \mathrm{pc}$ away. As an example, an error of only $0.05 \mathrm{mag}$ in $A_{V}$ when $T_{e}=10000 \mathrm{~K}$ gives a $3 \%$ error in $T_{e}$; more for hotter stars. Obviously this effect is greatest for hot stars due to their greater blue flux and tendency to be further away, and it may place the ultimate limit on how well we can calibrate their $T_{e}$ values. It is also a problem even for cool giants, however, as they also tend to be at large distances.

\subsection{LIMB DARKENING}

The $\theta$ measured by interferometry is not a true size, but that of an equivalent uniform disk (Davis, this volume). Model atmospheres are needed to provide a correction for limb darkening. The correction varies with wave- 
length and stellar class, being $\sim 3 \%$ for $\mathrm{G} / \mathrm{K}$ giants in the infra-red, and $\sim 10 \%$ for B main sequence stars in the blue. Working in the red reduces the correction, and thus hopefully makes it more reliable, but also reduces the resolution of the interferometer, reducing the coverage and accuracy of the $\theta$ measurements. The errors in the correction will usually be small enough to have a negligible effect on the accuracy of $T_{e}$.

\subsection{ATMOSPHERIC EXTENSION}

A problem related to but separate from limb darkening is the extension of giant star atmospheres. Scholz (this volume) gives a detailed appraisal of the difficulties this raises for stellar atmospheres. The effect is that angular sizes change with measurement wavelength (Quirrenbach et al., 1993), so care must be taken to ensure that enough information is gathered to characterise these changes and account for them in the analysis to obtain $T_{e}$. Measurements at one wavelength, even a continuum one are not enough.

\section{Summary}

At present the best interferometrically determined angular diameters combined with the best flux measurements can give effective temperatures to $1-2 \%$ accuracy. The available angular sizes limit the coverage for types of star, but this is due to the limitations of currently operating interferometers. The next generation of interferometers will greatly improve this situation. There is still room for improvement in the accuracy of flux calibrations and spectrophotometry, particularly in the UV and IR. Problems with these will continue to limit the accuracy of $T_{e}$ determinations for stars hotter than $\mathrm{A}$ and cooler than $\mathrm{G}$ in the near future.

\section{References}

Blackwell, D.E, Lynas-Gray, A.E. \& Petford, A.D. (1991), Astron. Astrophys., 245, 567.

Booth, A.J., Davis, J. \& Bessell, M. (1997), Proceedings of Science with the VLTI, Ed. Paresce, $F$., in press.

Code, A.D., Davis, J., Bless, R.C. \& Hanbury Brown, R. (1976), Astrophys. J., 203, 417.

Cohen, M., Walker, R.G., Barlow, M.J. \& Deacon, J.R. (1992), Astron. J., 104, 1650.

Di Benedetto, G.P. \& Rabbia, Y. (1987), Astron. Astrophys., 188, 114.

Dyck, H.M., Benson, J.A., van Belle, G.T. \& Ridgway, S.T. (1996), Astron. J., 111, 1705.

Habets, G.M.H.J. \& Heintze, J.R.W. (1981), Astron. Astrophys., 46, 193.

Hutter, D.J., Mozurkewich, D., Simon, R.S., Colavita, M.M. Pan, X.P., Shao, M., Hines, B.E., Staelin, D.H., Hershey, J.L., Hughes, J.A. \& Kaplan, G.H. (1989), Astrophys $J ., 340,1103$.

Petford, A.D., Blackwell, D.E., Booth, A.J., Haddock, D.J., Leggett, S.K., Mountain, C.M., Selby, M.J. \& Arribas, S. (1988), Astron. Astrophys., 203, 341.

Quirrenbach, A., Mozurkewich, D., Armstrong, J.T., Buscher, D.F. \& Hummel, C.A. (1993), Astrophys. J., 406, 215. 